\title{
Intercellular transport of RNA can limit heritable epigenetic changes
}

\author{
Authors: Nathan Shugarts ${ }^{1}$, Andrew L. Yi ${ }^{1}$, Winnie M. Chan ${ }^{1}$, Julia A. Marré ${ }^{1}$, Aishwarya \\ Sathya $^{1}$, and Antony M. Jose ${ }^{1 *}$
} Affiliations:

${ }^{1}$ Department of Cell Biology and Molecular Genetics, University of Maryland, College Park, MD 20742, USA.

*Correspondence to: amjose@umd.edu

\begin{abstract}
RNAs in circulation carry sequence-specific regulatory information between cells in animal, plant, and host-pathogen systems. Double-stranded RNA (dsRNA) delivered into the extracellular space of the nematode $C$. elegans accumulates within the germline and reaches progeny. Here we provide evidence for spatial, temporal, and substrate specificity in the transport of dsRNA from parental circulation to progeny. Temporary loss of dsRNA transport resulted in the persistent accumulation of mRNA from a germline gene. The expression of this gene varied among siblings and even between gonad arms within one animal. Perturbing RNA regulation of the gene created new epigenetic states that lasted for many generations. Thus, one role for the transport of dsRNA into the germline in every generation is to limit heritable changes in gene expression.
\end{abstract}

One Sentence Summary: RNA from parental circulation reduces heritable changes in gene expression.

Main text: RNAs released into circulation can act as intercellular messages that are used for gene regulation in distant cells. Specific examples include secretion of exosomal small RNAs in response to pathogenic fungal infection in Arabidopsis (1), virus-like proteins with their coding mRNAs in developing Drosophila (2) and mice (3), microRNAs from adipose tissue in mice (4), and small RNAs from the epididymis in mice (5-8). Such extracellular RNAs have also been 
detected in humans, but their roles in gene regulation remain unclear despite their use as a diagnostic tool for diseases (reviewed in (9)). Furthermore, the recent development of doublestranded RNA (dsRNA)-based drugs (reviewed in (10-11)) that can silence genes of matching sequence through RNA interference (12) has heightened interest in understanding the import of dsRNA into cells. A conserved dsRNA-selective importer, SID-1 (13-15), is required for the import of extracellular dsRNA into the cytosol of any cell in the nematode $C$. elegans. SID-1 has two homologs in mammals - SIDT1 and SIDT2. Although entry of ingested dsRNA into cells through SIDT1 (16), which can enhance dsRNA uptake when overexpressed in vitro (17), and entry of viral dsRNA through SIDT2 (18) have been reported in mice, alternative roles for these mammalian homologs in the uptake of cholesterol have also been proposed (19).

Secretion of dsRNA from C. elegans tissues that express dsRNA has been inferred based upon the SID-1-dependent silencing of matching genes in other tissues $(13,20)$. Secreted dsRNA from neurons can silence genes of matching sequence in most somatic cells (21) and within the germline (22). Extracellular dsRNA delivered into parental circulation by injection or ingestion also enters the germline and can cause silencing of matching genes in progeny $(12,23-26)$. Such intergenerational transport of RNA is an attractive mechanism for explaining gene-specific effects in progeny that could occur in response to changes in somatic tissues of parents. However, which conditions induce transport of dsRNA into the germline, when during development this transport occurs, and what the regulatory consequences are for such intercellular transport of dsRNA are all unknown.

Here we use oxidative damage in neurons expressing dsRNA or exposure to bacteria expressing dsRNA to demonstrate that dsRNA from parental circulation causes maximal silencing of germline gene expression during later development and in the proximal germline. 
Entry into the parental germline and subsequent transport in progeny occurs through two different intergenerational routes with distinct substrate specificities. Loss of dsRNA import through SID-1 alters the expression of a germline gene, inducing large changes in expression that can persist for many generations despite the restoration of dsRNA transport in descendants. The expression of this gene can vary between gonad arms and perturbing its RNA regulation can result in either reduced or increased expression. These changes in gene expression last for many generations, suggesting that loss of dsRNA transport induces new heritable epigenetic states.

\section{Oxidative damage in neurons expressing dsRNA enhances silencing in the germline by neuronal dsRNA}

To modulate the secretion of dsRNA from somatic cells into parental circulation during development, we adapted an approach for damaging somatic cells (27). Specifically, we generated animals that express the mini singlet oxygen generator (miniSOG) protein in neurons and exposed them to blue light. While animals expressing miniSOG from a single-copy transgene did not show an appreciable defect when compared with wild-type animals, those expressing miniSOG from a multi-copy transgene were paralyzed (Fig. S1A and S1B, top) and had visibly damaged neurons (Fig. S1B, bottom). Using this system, we induced oxidative damage in the neurons of animals that expressed dsRNA under the control of a neuronal promoter and evaluated silencing of target genes with matching sequence expressed in other tissues (Fig. 1A). By exposing animals to blue light for 60 minutes at different times during development (Fig. S1C), we observed SID-1-dependent enhancement in the silencing of the hypodermal gene bli- 1 in the adult stage by neuronal bli-1-dsRNA, with maximal silencing when oxidative damage occurred during mid-to-late larval development (Fig. S1D, light exposure from 42 to 66 hours post L4-stage of parent; Fig. S1E, 2 -fold increase from $14.9 \%$ to $29.1 \%$ in a 
background with enhanced RNA interference (eri-1(-)) and $\sim 6$-fold increase from $\sim 1.6 \%$ to $\sim 9.8 \%$ in a wild-type background). A similar period of maximal SID-1-dependent enhancement of silencing was also observed when neurons expressing $g f p$-dsRNA were damaged and silencing of a two-gene operon that expresses two fluorescent proteins, mCherry::H2B and GFP::H2B, in the germline was measured (Fig. 1B, Fig. 1C, Fig. 1D, Fig. S1F - 48 to 60 hours post L4-stage of parent, sid-1(-) allele (jam80[non]) depicted in Fig. S2). While silencing of $g f p:: h 2 b$ was observed throughout the germline, silencing of the other cistron $m C h e r r y:: h 2 b$ was often restricted to regions of the germline. Silencing of $m$ Cherry: $: h 2 b$ was most frequent in the proximal germline and was not observed in any other region without silencing in the proximal germline (proximal germline - 57\%, distal germline - 47\%, sperm - 29\%, Fig. 1D), likely due to reduction of $m$ Cherry: $: h 2 b:: g f p:: h 2 b$ pre-mRNA (28). The pattern of $m$ Cherry $:: h 2 b$ silencing is similar to the spatial pattern observed for the RME-2-dependent entry of dsRNA delivered into the parental circulation (25) and is consistent with the pattern of mRNA degradation in the germline by extracellular dsRNA (29).

These results suggest two insights into the transport of dsRNA from neurons to other tissues: (1) oxidative damage of neurons during particular periods in development increases the amount of dsRNA and/or changes the kinds of dsRNA in circulation either because of specific enhancement of secretion or nonspecific spillage; and (2) there is a preference for the entry of neuronal dsRNA into the proximal germline. These temporal and/or spatial preferences for silencing could be because of unknown characteristics of the exported neuronal dsRNA (e.g., modifications, lengths, structures, etc.) that influence import or subsequent silencing - a hypothesis that is also supported by the different requirements for silencing by neuronal $g f p$ - 
dsRNA compared to other sources of $g f p$-dsRNA (21). Alternatively, these preferences could reflect universal constraints for any extracellular dsRNA in C. elegans.

\section{Requirements for the entry of extracellular dsRNA into the germline vary during}

\section{development}

Another convenient method for the delivery of extracellular dsRNA into C. elegans at various times during larval development is the expression of dsRNA in the bacteria that worms ingest as food (23). To determine when ingested dsRNA can enter the germline and cause silencing, we exposed developing animals with a ubiquitously expressed protein (GTBP-1) tagged with GFP to bacteria that express $g f p$-dsRNA. Silencing was detectable within the germline from the second larval stage (L2) onwards (Fig. 1E, Fig. S3A), but exposure to ingested dsRNA beyond the fourth larval stage (L4) (Fig. 1F) or, alternatively, injection of dsRNA into the 1-day old adult germline (Fig. S3B) was required for silencing in the germline of 3-day old adults. The need for exposure to dsRNA during late development to observe persistent silencing suggests recovery of expression within the germline despite detectable silencing until the L4stage. Combined with the need for exposure to dsRNA after the L4 stage for silencing in progeny (25-26), these observations suggest that heritable RNA silencing is not effectively initiated during early development of the germline despite dsRNA entry and subsequent silencing. However, a 24-hour pulse of dsRNA exposure beginning at the L4 stage was sufficient for heritable silencing (Fig. S4A) (25). This early window for heritable silencing likely relies on entry of dsRNA into the proximal germline because (1) silencing of a somatic gene in progeny after parental ingestion of dsRNA required RME-2 (Fig. S4A), which is enriched in the proximal germline (Fig. S4B) (30); and (2) some $g t b p-1: \because g f p$ animals exposed to $g f p$-dsRNA until the first day of adulthood showed selective silencing in the proximal germline (Fig. S3C). 
Together, these results reveal three periods of germline development that can be broadly distinguished based on silencing in response to ingested and neuronal dsRNA: (1) from the first larval to the third larval stage when exposure to dsRNA does not result in maximal silencing within the germline in adults; (2) from the fourth larval stage to early adulthood when entry of dsRNA primarily occurs in the proximal germline through RME-2; and (3) later adulthood when entry can be independent of RME-2 (Fig. S4A) (26) and germline silencing by ingested dsRNA is maximal.

\section{Different forms of dsRNA from parental circulation require different members of the transport pathway in developing progeny for silencing}

When exposing animals to dsRNA expressed in bacteria, the forms of dsRNA made and processed in bacteria cannot be easily controlled. Microinjection of dsRNA into the pseudocoelom $(12,25)$ provides a way to deliver particular forms of extracellular dsRNA into $C$. elegans, but can be most easily performed only using L4-staged and adult animals. We examined differences, if any, in the entry of in vitro transcribed dsRNA into the germline during these two stages as evidenced by silencing in progeny. Silencing was comparable regardless of whether wild-type or rme-2(-) parents were injected as L4-staged or adult animals (Fig. 2A, Fig. S4C, left; also reported for adults in (26)), although a weak requirement for RME-2 was discernable when lower concentrations of dsRNA were used (Fig. S4C, right). The difference in RME-2 requirement between ingested dsRNA and injected dsRNA could reflect parental circulation accumulating different amounts of dsRNA (e.g., more upon injection than upon ingestion) and/or different kinds of dsRNA (e.g., because of modifications in bacteria or upon transit through the intestine). However, these possibilities could not be easily distinguished because sensitive northern blotting (31) revealed that both bacterial and in vitro transcribed dsRNA consist of a 
complex mix of dsRNAs (Fig. S4D, Fig. S4E, Fig. S4F; consistent with (32-33)), hereafter called mixed dsRNA. In contrast, when synthesized $g f p$-dsRNA of a defined length (50 bp) with a fluorescent label was injected into circulation in adult animals, no entry into the germline was observed in the absence of RME-2 (25). We found that silencing of unc-22 in progeny by similarly synthesized but unlabeled 50-bp unc-22-dsRNA with a 5'-OH delivered into parental circulation also showed a strong requirement for RME-2 compared to mixed dsRNA (Fig. 2A). Further comparison between the two forms of dsRNA revealed that silencing in progeny by 50bp dsRNA injected into parental circulation was detectably less efficient in somatic cells (Fig. 2B, Fig. S5A, Fig. S5B, left), even when $\sim 14 \mathrm{X}$ more 50-bp dsRNA was delivered into parental circulation (Fig. S5B, right), and was also less efficient in the germline (Fig. 2B, Fig. S5A, Fig. S5C). Given that both 50-bp dsRNA and mixed dsRNA rely on the nuclear Argonaute HRDE-1 (34) for silencing within the germline (Fig. S5A, Fig. S5C) and can silence independent of the nuclear Argonaute NRDE-3 (28) in somatic cells (Fig. S5A, Fig. S5C), the observed difference in the extent of silencing could be the result of differences in the stability and/or intergenerational transport of 50-bp dsRNA versus mixed dsRNA. One relevant feature shared by mixed dsRNA generated in bacteria or in vitro, in addition to the diversity of lengths (Fig. S4), is that both forms contain 5' triphosphates. In support of the impact of 5' phosphates on transport and/or silencing, addition of 5' monophosphates to synthesized 50-bp dsRNA injected into parental circulation reduced the dependence on RME-2 for silencing in progeny (Fig. S4G, Fig. S4H). Thus, the requirements for entry into the germline and subsequent silencing vary for different lengths and/or chemical forms of dsRNA.

Fluorescently labeled 50-bp dsRNA delivered into parental circulation localized within intestinal cells in progeny (Fig. 2C, top left), as has been observed for vitellogenin proteins (35) 
and fluorescent dyes (36). Accumulation of fluorescently-labeled dsRNA was also detected at the apical membrane of the intestine, which could reflect exocytosis of dsRNA into the lumen of developing intestinal cells. However, separation of the fluorescent label from dsRNA catalyzed by cellular enzymes cannot be excluded. Therefore, to dissect differences, if any, between the transport of short dsRNA (synthesized 50-bp with 5'OH) and mixed dsRNA (mixture transcribed in vitro using $\sim 1 \mathrm{~kb}$ DNA template) we injected unc-22-dsRNA into animals with mutations in genes that play roles in the import of dsRNA. We found that maternal SID-1 was required for silencing by short dsRNA in progeny (Fig. 2C, bottom, left bars), suggesting that the SID-1dependent entry of short dsRNA into the cytosol likely occurs in the injected parent or during early development in progeny. Uptake of dsRNA from the intestinal lumen requires SID-2, a transmembrane protein located in the apical membranes of intestinal cells (37-38). We found that SID-2 was not required for most silencing in progeny by short or mixed dsRNA injected into parental circulation (Fig. 2C, top right and bottom). Exit of dsRNA from intracellular vesicles requires SID-5, a transmembrane protein located in endolysosomal membranes (39). Silencing in wild-type animals was comparable to silencing in sid-5(-) animals (Fig. 2C, top right). However, when animals that lacked SID-1 were injected, SID-5 was required in progeny for silencing by mixed dsRNA from parental circulation (Fig. 2C, bottom, right bars; as also reported in (26)). Since dsRNA is expected to be present in vesicles upon entry through RME-2 in the absence of SID-1 (25-26), this observation suggests that SID-5 is required for the release of mixed dsRNA from inherited vesicles in progeny.

In summary, extracellular dsRNA can enter the germline in parents and be transmitted to progeny through two routes with different substrate selectivity. One route is preferentially used by short dsRNA and relies on RME-2-mediated endocytosis of dsRNA into oocytes, where early 
exit from vesicles is required for silencing in progeny as evidenced by the need for maternal

SID-1 (Fig. 2D, blue). The other route appears to exclude short dsRNA, but allows mixed dsRNA entry into the cytosol in the parental germline through SID-1 and exit from inherited vesicles in progeny through a process that requires both zygotic SID-1 and SID-5 (Fig. 2D, grey) (26).

\section{Expression of SID-1 is consistent with a role in intergenerational transport of extracellular dsRNA but could be differentially regulated across cell types}

Analysis of dsRNA transport into the germline and to progeny suggests developmental variation in the expression pattern of SID-1. Previous attempts at observing SID-1 localization relied on multi-copy transgenes (13), which can become silenced within the germline (40) and could produce a variety of tagged and untagged proteins (41). When using multi-copy transgenes to express a SID-1 fusion protein tagged at the C-terminus with DsRed or GFP (Fig. S6A) under the control of a promoter that drives expression within body-wall muscles, we observed intracellular localization of SID-1::DsRed or SID-1::GFP (Fig. S6B, top) along with rescue of gene silencing by ingested dsRNA in body-wall muscles (Fig. S6B, bottom). However, similar tagging to express SID-1 fusion proteins from either a single-copy transgene expressed in the germline (SID-1::DsRed) or the endogenous locus (SID-1::wrmScarlet) did not enable gene silencing by ingested dsRNA (Fig. S6C), suggesting that the C-terminal fusions of SID-1 were likely non-functional and that apparent function when using multi-copy transgenes reflects production of untagged variants. In support of our rationale, a recent prediction of SID-1 structure (42) suggests that the C-terminus is sequestered, a feature that may be disrupted by the addition of C-terminal fluorophores, potentially leading to misfolded proteins that are degraded. Consistently, we found that internal tagging of the sid-1 gene using Cas9-mediated genome 
editing to express SID-1::mCherry (Fig. 3A) resulted in a fusion protein with detectable function (Fig. 3B, Fig. S6D). Therefore, we analyzed fluorescence from this fusion protein expressed from the endogenous locus under the control of native regulatory sequences (Fig. 3C, Fig. 3D, Fig. S6E, Fig. S6F, Fig. S6G). Fluorescence from SID-1::mCherry progressively increased during development with tissue-specific enrichments in the developing embryo (Fig. 3C, left, Fig. S6G), becoming ubiquitous in hatched L1 larvae (Fig. 3C, middle). SID-1::mCherry was not easily detectable in the germline during larval development (Fig. 3C, middle and right), but was visible in the proximal and distal regions of the adult germline (Fig. 3D). Similarly, endogenous RME-2 was most abundant in the proximal oocytes of the adult germline (Fig. S4B) (30). These expression patterns are consistent with the entry of most dsRNA from circulation of adult parents into the proximal germline (25) and the activity of transport mechanisms in developing embryos (Fig. 2).

To determine if acute induction of SID-1 expression would be sufficient for the import of dsRNA into different cell types, we engineered the endogenous sid-1 gene to transcribe a fusion transcript with an aptamer-regulated ribozyme (Fig. S7A, left) that cleaves itself when not bound to tetracycline (Fig. S7A, right) (based on (43)). Exposing these animals to tetracycline enabled silencing by dsRNA in somatic tissues (hypodermis: Fig. S7B, left; body-wall muscles: Fig. S7B, right) but not in the germline (Fig. S7C, Fig. S7D, Fig. S7E, Fig. S7F), indicative of stabilization of sid-1 mRNA, production of SID-1 protein, and subsequent dsRNA import in somatic cells but not in the germline. Yet, similar tagging of the ubiquitously expressed gene $g t b p-1: \because g f p$ results in detectable rescue of expression within the germline by tetracycline (Fig. S7G). A possible explanation for the poor rescue of SID-1 activity within the germline is that post-transcriptional mechanisms targeting sid-1 mRNA in the germline but not the soma interfere with tetracycline- 
dependent stabilization of the sid-1 transcript (e.g., piRNA-based regulation of sid-1 mRNA (4445)).

Further improvements in tagging SID-1 protein, potentially guided by structure, and sid-1 transcript, potentially guided by post-transcriptional regulatory interactions, could enable deeper analysis of dsRNA transport between cells. Nevertheless, the developmentally regulated expression observed for both SID-1 and RME-2 in the germline is consistent with intergenerational or transgenerational effects regulated by dsRNA from parental circulation after development of the adult germline.

\section{Temporary loss of SID-1 results in a large increase in mRNA from a germline gene that lasts for many generations}

To understand how transport of extracellular dsRNA into the germline might be used for endogenous gene regulation across generations, we searched for sid-1-dependent changes in gene expression that could be heritable (Fig. 3, Fig. S2, Fig. S8, Fig. S9, Fig. S10). We initially analyzed polyA+ RNAs extracted from wild-type, sid-1(qt9), sid-1(tm2700), and sid-1(tm2700); tmIs1005[sid-1(+)] animals and found that comparisons between samples with similar genetic backgrounds did not result in a consistent list of SID-1-dependent genes (Fig. S8). Strains with similar genotypes $($ sid-1(+) or sid-1(-)) did not cluster together when using principal component analysis (Fig. S8A), suggesting that other differences in genetic background could obscure or misrepresent differences between sid-1(+) and sid-1(-) animals. To ameliorate this problem we used Cas9-mediated genome editing to delete the entire sid-1 coding sequence ( $\mathrm{del})$ or introduce a nonsense mutation (non) in cohorts of the same wild-type animals. When comparing polyA+ RNA from this wild type with that of the newly generated sid-1(jam113[del]) (Fig. 3A, Fig. 3B, Fig. S9A) or sid-1(jam80[non]) (Fig. 3A, Fig. 3B, Fig. 3E) animals, we found that 26 genes were 
significantly $(q<0.05)$ misregulated in sid-1(jam113[del]) (Fig. S9B) and 6 in sid-

1(jam80[non]) (Fig. 3F, left), both including sid-1. However, the most upregulated gene in sid1(jam113[del]), F14F9.5, was likely perturbed as a consequence of disrupting regulation near the sid-1 locus through deletion of DNA and not because of loss of SID-1 function because this change was only observed in the deletion mutant sid-1(jam113[del]) and not in the newly generated nonsense mutant sid-1(jam80[non]) (Fig. S9D, left), despite both mutants being equally defective for silencing by ingested dsRNA (Fig. 3B). Nevertheless, we could detect two genes that were upregulated in both sid-1(jam113[del]) and sid-1(jam80[non]) animals (red in Fig. 3F, left, Fig. S9B): the identical loci W09B7.2/F07B7.2 (Fig. S9D, middle) and Y102A5C.36 (Fig. S9D, right) - each expressed within the germline (Fig. S10, left) and regulated by endogenous small RNAs (Fig. S10, middle and right). While spliced mRNA levels measured at a later generation using RT-qPCR demonstrated that both transcripts were upregulated in sid1(jam80[non]) animals compared to wild-type animals as expected (Fig. 3G), no upregulation was detectable in sid-1(jam113[del]) animals (Fig. S9C), potentially because of complex effects caused by deleted DNA (e.g., F14F9.5 overexpression) that are independent of SID-1 function. To determine if changes in W09B7.2/F07B7.2 and Y102A5C.36 expression were heritable, we reverted the sid-1 nonsense mutation to wild-type sequence using Cas9-mediated genome editing. This immediately restored most silencing by ingested dsRNA, reaching wild-type levels of SID-1 function within two generations (Fig. S11, Fig. 3B) with concomitant recovery of sid-1 mRNA to wild-type levels (Fig. 3G, left). In contrast, changes in both W09B7.2/F07B7.2 and Y102A5C.36 expression persisted (Fig. 3F, right) even after a year of passaging revertants (i.e., after $>100$ generations, Fig. 3G, middle and right). Since the change in W09B7.2/F07B7.2 
mRNA was large (Fig. 3G, middle, 8 -fold), we focused on heritable changes in the expression of this gene in this study and hereafter refer to this sid-1-dependent gene (sdg) as sdg-1.

\section{Expression of $s d g-1$ can vary within an animal and perturbing its RNA regulation creates new epigenetic states that last for many generations}

To facilitate analysis of SDG-1 expression, we tagged both loci that express SDG-1 with mCherry coding sequences lacking piRNA-binding sites (mCherry $\Delta p i)$ (46-47) (Fig. S12A, Fig. S12B), thereby preventing possible silencing of $m$ Cherry as a foreign sequence. Consistently, expression of SDG-1::mCherry was detectable by fluorescence microscopy and remained detectable for many generations (Fig. 4A, Fig. S12C). The expression of sdg-1::mCherry $\Delta p i$ mRNA was $~ 16$-fold higher than $s d g-1$ mRNA (Fig. S12D), potentially because of the additional introns included in mCherry $\Delta p i$ (48-49) and/or other unknown factors. Fluorescence from SDG1::mCherry was observed in the germline of adult animals (Fig. 4A, top left), in early embryos (Fig. 4A, right and bottom left), and in potentially extracellular punctae near the proximal germline (Fig. 4A, top left and right). Intriguingly, SDG-1::mCherry dynamically entered the nucleus from the cytoplasm before fertilization (Fig. 4A, right, Movie S1) and before early cell divisions in the developing embryo (Fig. 4A, bottom left, Movie S2, Movie S3). Additional recent observations suggest that SDG-1 is a regulated protein that could itself play a role in RNA-based regulation within the germline: (1) the dynamic subcellular localization of the SDG1 protein in the -1 oocyte is similar to that of the essential Argonaute CSR-1b (50); (2) the SDG1 protein interacts with PID-2 (51) and potentially DEPS-1 (52) - two proteins with roles in heritable piRNA-induced silencing; and (3) loss of the germline Argonaute HRDE-1 results in upregulation of transcripts from a region that includes the $s d g-1$ gene (53). Thus, one hypothesis suggested by the large and persistent change in $s d g-1$ expression upon loss of SID-1 is that 
extracellular dsRNA-based regulation of $s d g-1$ protects it from heritable epigenetic change initiated by other mechanisms within the germline.

The proposed susceptibility of $s d g-1$ expression to heritable epigenetic change is supported by four lines of evidence. One, simply mating animals that express SDG-1::mCherry with wild-type animals resulted in heritable changes along lineages that express $s d g$ 1::mCherry $\Delta p i$ mRNA or that express $s d g-1$ mRNA (Fig. 4B, Fig. S13). Two, Cas9-mediated genome editing of genes required for dsRNA import or subsequent silencing (Fig. 4C), but not of unrelated genes (Fig. S14), resulted in some isolates that showed dramatically reduced or increased expression (Fig. 4C). While possible mechanisms mediating increased expression are unclear, decreased expression could be mediated by piRNAs that target $s d g-1$ since expression in both isolates lacking DEPS-1, a protein required for piRNA-mediated silencing (54-55), showed increased expression (Fig. 4C). Three, isolating siblings led to lineages with distinct levels of $s d g-1$ expression in some cases (compare sibling lineages in Fig. 4B and in Fig. S14). Four, many animals showed dramatic variation in SDG-1::mCherry expression between their two gonad arms (Fig. 4D). The two identical loci referred to as $s d g-1$ are part of a $\sim 40-\mathrm{kb}$ duplicated region (Fig. S15), which could be a contributing feature for the observed stochasticity as suggested by RNA silencing of multi-copy genes (41).

While loss of SID-1 in otherwise wild-type animals led to a persistent increase in $s d g-1$ mRNA in our earlier RNA-seq and RT-qPCR experiments (Fig. 3), loss of SID-1 in $s d g$ 1::mCherry $\Delta p i$ animals led to a decrease or increase in SDG-1::mCherry in separate lineages of newly generated sid-1(-) isolates (Fig. 4C, e.g., compare sid-1(jam150) and sid-1(jam177)), potentially because of differences in the levels of $s d g-1$ expression before loss of SID-1. Once downregulated, reduced levels of SDG-1::mCherry persisted across generations after restoration 
of dsRNA transport (Fig. 4E), just as the upregulation of untagged $s d g-1$ mRNA also persisted (Fig. 3F, Fig. 3G).

Together, these results suggest that one or both $s d g-1$ loci are subject to heritable changes upon loss of SID-1-mediated gene regulation and that the direction of change might depend upon the levels of $s d g-1$ mRNA. Thus, one function of SID-1, and potentially dsRNA(s) that enter cells through SID-1, is to reduce stochastic initiation of heritable epigenetic changes in gene expression within the germline.

\section{Discussion}

We found that germline entry of dsRNA released from neurons upon oxidative damage and germline entry of ingested dsRNA occurs with spatiotemporal specificity. Such uptake of extracellular dsRNA from parental circulation and subsequent trafficking in progeny can occur through at least two routes that select for different forms of dsRNA. When the entry of all endogenous dsRNA into the cytosol is blocked, large increases or decreases in the expression of a germline gene can be observed in different animals that are genetically identical. These new expression states can persist for many generations despite restoration of dsRNA transport, suggesting a role for intercellular gene regulation by dsRNA in preventing heritable changes in gene expression.

\section{Oxidative damage and the physiological conditions that favor secretion of dsRNA}

The physiological conditions that promote secretion of dsRNA are not known. Our discovery that oxidative damage of neurons can enhance the secretion of dsRNA suggests that disruption of cell structures by oxidative damage (e.g., membrane integrity) or initiation of cellular processes that repair oxidative damage (e.g., through ejection of damaged 
macromolecules (56)) also promote the release of dsRNA. Alternatively, damage-induced increase in the accumulation of dsRNA through indirect mechanisms could also explain the results observed. Pathologies of the central nervous system in humans, including cancer, stroke, multiple sclerosis, neurodegenerative disease, and brain injury, have been associated with extracellular RNAs detected in circulation (reviewed in (57)), although their origins and regulatory consequences, if any, remain unknown. The gene regulatory effects of neuronal dsRNA released upon oxidative damage of neurons provide convenient readouts that can be analyzed to understand neuronal damage and its consequences in animals.

\section{Specificity in the intergenerational transport of extracellular dsRNA}

The trafficking of extracellular dsRNA from parent to progeny has spatial specificity, as evidenced by more silencing within the proximal germline (Fig. 1), temporal specificity, as evidenced by the need for dsRNA beyond the fourth larval stage (Fig. 1) (25-26), and substrate specificity, as evidenced by the differential requirements for 50-bp dsRNA with 5'-OH versus a mix of longer dsRNAs with 5' triphosphates (Fig. 2). One possible explanation for these constraints could be that proteins mediating dsRNA transport differ in their availability during development and in their affinities for different substrates. For example, SID-1, which was not detected in the developing larval germline but was detected in the adult germline (Fig. 3), has an extracellular domain that binds dsRNA (58) and could prefer dsRNA molecules with 5' phosphates. Although the selectivity uncovered here could apply to all dsRNA delivered into the extracellular space of $C$. elegans from any source, the chemistry of the delivered dsRNA could be modified by as yet unidentified enzymes in vivo to overcome these requirements. Tracking labeled dsRNA with diverse chemistries from parental circulation to progeny could allow 
correlation of differences observed in progeny silencing to differences in intergenerational trafficking.

\section{SID-1-dependent defects in gene regulation}

The germline is a major site of dsRNA import in C. elegans as evidenced by the expression of SID-1 in the germline (Fig. 3), heritable misregulation of germline genes in sid-1() animals (Fig. 3, Fig. 4), and accumulation of fluorescently-labeled dsRNA from the extracellular space in the germline (25-26). As a result, sid-1(-) animals could have a defect in the germline that is detectable only under conditions that promote dsRNA transport (e.g., oxidative damage). Multiple physiological defects in the germline and soma of sid-1(-) animals have been reported, but have not been widely reproduced, have only been characterized within single generations, and have not been attributed to any specific sid-1-dependent gene(s). These include defects in animals exiting the dauer stage (59-60), in animals exposed to pathogenic $P$. aeruginosa (61-63), in animals exposed to odor (64), and in intestinal cells that develop in the presence of a multi-copy transgene (65). RNA-seq experiments in this study suggest that genetic background-dependent changes can obscure genuine sid-1-dependent changes (Fig. S8, Fig. S9), raising caution in the interpretation of putative sid-1-dependent defects. Comparing sid-1 mutants generated using genome editing with animals in which the mutated sequence has been reverted to wild-type sequence in the same genetic background could provide a firmer basis for the identification of sid-1-dependent processes.

\section{Buffered RNA regulation in the germline as a guard against heritable epigenetic changes}

A role for SID-1 in preventing heritable epigenetic changes in the expression of the endogenous gene $s d g-1$ is unexpected in light of previous demonstration that the import of dsRNA into the germline through SID-1 can initiate heritable RNA silencing of a single-copy 
transgene (22). This difference can be understood by considering that the regulatory context of a gene could dictate its response to dsRNA exposure. In support of this idea, targeting a few genes containing matching sequences with the same extracellular dsRNA revealed that while most genes recover from RNA silencing, some are susceptible to stable RNA silencing (47). Consistently, the expression of $s d g-1$ is extraordinarily susceptible to heritable epigenetic change, precluding typical analysis of genetic requirements through mating to mutant backgrounds (Fig. 4B) and necessitating Cas9-mediated genome editing (Fig. 4C).

In general, genes expressed within the germline are likely regulated by positive feedback loops that continually produce factors for maintaining germline immortality and for preserving form and function across generations (66-67). Thus, germline genes could be particularly vulnerable to heritable epigenetic changes, where deviations in the expression levels of a gene that is regulated by or is part of such feedback loops have the potential to become permanent in descendants. Perturbations in $s d g-1$ expression by multiple methods in this study suggest that $s d g-1$ is part of a regulatory architecture that is susceptible to heritable epigenetic change. To buffer against such changes, levels of gene expression would need to be maintained within a particular range for a given regulatory context. We propose that expression of $s d g-1$ is maintained by dsRNA imported through SID-1 and downstream small RNAs, and speculate that one role for extracellular RNAs that enter germ cells in other systems (e.g., tRNA fragments in mammals $(5-6,8))$ could be to similarly buffer against heritable changes in gene expression.

Acknowledgements: We thank Mary Chey, Samiha Tasnim, and Daphne Knudsen for comments on the manuscript; the Caenorhabditis elegans Genetic Stock Center, the Seydoux laboratory (Johns Hopkins University) and the Hunter laboratory (Harvard University) for worm strains; Quentin Gaudry for help in creating our optogenetics apparatus; the Andrews laboratory for use of 
a confocal microscope; Amy Beaven and the Imaging Core Facility for temporary use of a Leica TCS SP8 DLS microscope with HyVolution; Lanelle Edwards, Rex Ledesma, Carlos Machado, and Omega Bioservices for help with RNA sequencing and analysis. Funding: This work was supported by UMD CMNS Dean's Matching Award for “Training Program in Cell and Molecular Biology" T32GM080201 to N.S. and in part by National Institutes of Health Grants R01GM111457 and R01GM124356 to A.M.J. Author contributions: N.S. and A.M.J designed the research. N.S., A.L.Y., W.M.C., and A.S. performed all experiments, and collected and analyzed data. N.S. and A.M.J. prepared the manuscript with contributions from all authors. Data and Materials availability: All data and code are available in the manuscript or the supplementary materials. RNA-seq data has been deposited to Gene Expression Omnibus (GEO) with the accession number GSE185385. 


\section{References and Notes:}

1. Q. Cai, L. Qiao, M. Wang, B. He, F. M. Lin, J. Palmquist, S. D. Huang, H. Jin, Plants send small RNAs in extracellular vesicles to fungal pathogen to silence virulence genes. Science $\mathbf{3 6 0}$, 1126-1129 (2018).

2. J. Ashley, B. Cordy, D. Lucia, L. G. Fradkin, V. Budnik, T. Thomson, Retrovirus-like Gag protein Arc1 binds RNA and traffics across synaptic boutons. Cell 172, 262-274 (2018).

3. E. D. Pastuzyn, C. E. Day, R. B. Kearns, M. Kyrke-Smith, A. V. Taibi, J. McCormick, N. Yoder, D. M. Belnap, S. Erlendsson, D. R. Morado, J. A. G. Briggs, C. Feschotte, J. D. Shephard, The neuronal gene Arc encodes a repurposed retrotransposon Gag protein that mediates intercellular RNA transfer. Cell 172, 275-288 (2018).

4. T. Thomou, M. A. Mori, J. M. Dreyfuss, M. Konishi, M. Sakaguchi, C. Wolfrum, T. N. Rao, J. N. Winnay, R. Garcia-Martin, S. K. Grinspoon, P. Gorden, C. R. Kahn, Adipose-derived circulating miRNAs regulate gene expression in other tissues. Nature 542, 450-455 (2017).

5. U. Sharma, C. C. Conine, J. M. Shea, A. Boskovic, A. G. Derr, X. Y. Bing, C. Belleannee, A. Kucukural, R. W. Serra, F. Sun, L. Song, B. R. Carone, E. P. Ricci, X. Z. Li, L. Fauquier, M. J. Moore, R. Sullivan, C. C. Mello, M. Garber, O. J. Rando, Biogenesis and function of tRNA fragments during sperm maturation and fertilization in mammals. Science 351(6271), 391-396 (2016).

6. Q. Chen, M. Yan, Z. Cao, X. Li, Y. Zhang, J. Shi, G.-H. Feng, H. Peng, X. Zhang, Y. Zhang, J. Qian, E. Duan, Q. Zhai, Q. Zhou, Sperm tsRNAs contribute to intergenerational inheritance of an acquired metabolic disorder. Science 351(6271), 397-400 (2016).

7. C. C. Conine, F. Sun, L. Song, J. A. Rivera-Pérez, O. J. Rando, Small RNAs gained during epididymal transit of sperm are essential for embryonic development in mice. Dev. Cell 46(4), 470-480.e3 (2018).

8. U. Sharma, F. Sun, C. C. Conine, B. Reichholf, S. Kukreja, V. A. Herzog, S. L. Ameres, O. J. Rando, Small RNAs are trafficked from the epididymis to developing mammalian sperm. Dev. Cell 46(4), 481-494.e6 (2018).

9. S. Das, Extracellular RNA Communication Consortium, K. M. Ansel, M. Bitzer, X. O. Breakefield, A. Charest, D. J. Galas, M. B. Gerstein, M. Gupta, A. Milosavljevic, M. T. McManus, T. Patel, R. L. Raffai, J. Rozowsky, M. E. Roth, J. A. Saugstad, K. Van KeurenJensen, A. M. Weaver, L. C. Laurent, The Extracellular RNA Communication Consortium: establishing foundational knowledge and technologies for extracellular RNA research. Cell 177, 231-242 (2019).

10. R. L. Setten, J. J. Rossi, S. P. Han, The current state and future directions of RNAi-based therapeutics. Nat. Rev. Drug Discov. 18, 421-446 (2019). 
11. B. Hu, L. Zhong, Y. Weng, L. Peng, Y. Huang, Y. Zhao, X.-J. Liang, Therapeutic siRNA: state of the art. Signal Transduct. Target Ther. 5(1), 101 (2020).

12. A. Fire, S. Xu, M. K. Montgomery, S. A. Kostas, S. E. Driver, C. C. Mello, Potent and specific genetic interference by double-stranded RNA in Caenorhabditis elegans. Nature 391(6669), 806-811 (1998).

13. W. M. Winston, C. Molodowitch, C. P. Hunter, Systemic RNAi in C. elegans requires the putative transmembrane protein SID-1. Science 295, 2456-2459 (2002).

14. E. H. Feinberg, C. P. Hunter, Transport of dsRNA into cells by the transmembrane protein SID-1. Science 301(5639), 1545-1547 (2003).

15. J. D. Shih, M. C. Fitzgerald, M. Sutherlin, C. P. Hunter, The SID-1 double-stranded RNA transporter is not selective for dsRNA length. RNA 15(3), 384-390 (2009).

16. Q. Chen, F. Zhang, L. Dong, H. Wu, J. Xu, H. Li, J. Wang, Z. Zhou, C. Liu, Y. Wang, Y. Liu, L. Lu, C. Wang, M. Liu, X. Chen, C. Wang, C. Zhang, D. Li, K. Zen, F. Wang, Q. Zhang, C.-Y. Zhang, SIDT1-dependent absorption in the stomach mediates host update of dietary and orally administered microRNAs. Cell Res. 31(3), 247-258 (2021).

17. T. A. Nguyen, B. R. C. Smith, K. D. Elgass, S. J. Creed, S. Cheung, M. D. Tate, G. T. Belz, I. P. Wicks, S. L. Masters, K. C. Pang, SIDT1 Localizes to Endolysosomes and Mediates Double-Stranded RNA Transport into the Cytoplasm. J Immunol. 202(12), 3483-3492 (2019).

18. T. A. Nguyen, B. R. C. Smith, M. D. Tate, G. T. Belz, M. H. Barrios, K. D. Elgass, A. S. Weisman, P. J. Baker, S. Preston, L. Whitehead, A. Garnham, R. J. Lundie, G. K. Smyth, M. Pellegrini, M. O'Keeffe, I. P. Wicks, S. L. Masters, C. P. Hunter, K. C. Pang, SIDT2 transports extracellular dsRNA into the cytoplasm for innate immune recognition. Immunity 47(3), 498509.e6 (2017).

19. K. M. Méndez-Acevedo, V. J. Valdes, A. Asanov, L. Vaca, A novel family of mammalian transmembrane proteins involved in cholesterol transport. Sci. Rep. 7(1), 7450 (2017).

20. A. M. Jose, J. J. Smith, C. P. Hunter, Export of RNA silencing from C. elegans tissues does not require the RNA channel SID-1. Proc. Natl. Acad. Sci. 106, 2283-2288 (2009).

21. S. Ravikumar, S. Devanapally, A. M. Jose, Gene silencing by double-stranded RNA from $C$. elegans neurons reveals functional mosaicism of RNA interference. Nucleic Acids Res. 47(19), 10059-10071 (2019).

22. S. Devanapally, S. Ravikumar, A. M. Jose, Double-stranded RNA made in C. elegans neurons can enter the germline and cause transgenerational gene silencing. Proc. Natl. Acad. Sci. 112, 2133-2138 (2015). 
23. L. Timmons, A. Fire, Specific interference by ingested dsRNA. Nature 395(6705), 854 (1998).

24. A. Grishok, H. Tabara, C. C. Mello, Genetic requirements for inheritance of RNAi in $C$. elegans. Science 287(5462), 2494-2497 (2000).

25. J. Marré, E. C. Traver, A. M. Jose, Extracellular RNA is transported from one generation to the next in Caenorhabditis elegans. Proc. Natl. Acad. Sci. 113, 12496-12501 (2016).

26. E. Wang, C. P. Hunter, SID-1 functions in multiple roles to support parental RNAi in Caenorhabditis elegans. Genetics 207, 547-557 (2017).

27. S. Xu, A. D. Chisholm, Highly efficient optogenetic cell ablation in C.elegans using membrane-targeted miniSOG. Sci. Rep. 6, 21271 (2016).

28. S. Guang, A. F. Bochner, D. M. Pavelec, K. B. Burkhart, S. Harding, J. Lachowiec, S. Kennedy, An Argonaute transports siRNAs from the cytoplasm to the nucleus. Science 321, 537$541(2008)$.

29. J. P. T. Ouyang, W. Zhang, G. Seydoux, Two parallel sRNA amplification cycles contribute to RNAi inheritance in C. elegans. bioRxiv: 10.1101/2021.08.13.456232 (2021).

30. B. Grant, D. Hirsh, Receptor-mediated endocytosis in the Caenorhabditis elegans oocyte. Mol. Biol. Cell 10(12), 4311-4326 (1999).

31. Y. S. Choi, L. O. Edwards, A. DiBello, A. M. Jose, Removing bias against short sequences enables northern blotting to better complement RNA-seq for the study of small RNAs. Nucleic Acids Res. 45, e87 (2017).

32. H. Tabara, E. Yigit, H. Siomi, C. C. Mello, The dsRNA binding protein RDE-4 interacts with RDE-1, DCR-1, and a DExH-box helicase to direct RNAi in C. elegans. Cell 109(7), 861-871 (2002).

33. N. Jain, L. R. Blauch, M. R. Szymanski, R. Das, S. K. Y. Tang, Y. W. Yin, A. Z. Fire, Transcription polymerase-catalyzed emergence of novel RNA replicons. Science $\mathbf{3 6 8}(\mathbf{6 4 8 7})$, eaay0688 (2020).

34. B. A. Buckley, K. B. Burkhart, S. G. Gu, G. Spracklin, A. Kershner, H. Fritz, J. Kimble, A. Fire, S. Kennedy, A nuclear Argonaute promotes multigenerational epigenetic inheritance and germline immortality. Nature 489, 447-451 (2012).

35. W. J. Sharrock, Yolk proteins of Caenorhabditis elegans. Dev. Biol. 96(1), 182-188 (1983).

36. O. Bossinger, E. Schierenberg, The use of fluorescent marker dyes for studying intercellular communication in nematode embryos. Int. J. Dev. Biol. 40(1), 431-439 (1996). 
37. W. M. Winston, M. Sutherlin, A. J. Wright, E. H. Feinberg, C. P. Hunter, Caenorhabditis elegans SID-2 is required for environmental RNA interference. Proc. Natl. Acad. Sci. 104, 10565-10570 (2007).

38. D. L. McEwan, A. S. Weisman, C. P. Hunter, Uptake of extracellular double-stranded RNA by SID-2. Mol. Cell 47, 746-754 (2012).

39. A. Hinas, A. J. Wright, C. P. Hunter, SID-5 is an endosome-associated protein required for efficient systemic RNAi in C. elegans. Curr. Biol. 22, 1938-1943 (2012).

40. W. G. Kelly, S. Xu, M. K. Montgomery, A. Fire, Distinct requirements for somatic and germline expression of a generally expressed Caenorhabditis elegans gene. Genetics 146(1), 227-238 (1997).

41. H. H. Le, M. Looney, B. Strauss, M. Bloodgood, A. M. Jose, Tissue homogeneity requires inhibition of unequal gene silencing during development. J. Cell Biol. 14(3), 319-331 (2016).

42. J. Jumper, R. Evans, A. Pritzel, T. Green, M. Figurnov, O. Ronneberger, K.

Tunyasuvunakool, R. Bates, A. Žídek, A. Potapenko, A. Bridgland, C. Meyer, S. A. A. Kohl, A. J. Ballard, A. Cowie, B. Romera-Paredes, S. Nikolov, R. Jain, J. Adler, T. Back, S. Petersen, D. Reiman, E. Clancy, M. Zielinski, M. Steinegger, M. Pacholska, T. Berghammer, S. Bodenstein, D. Silver, O. Vinyals, A. W. Senior, K. Kavukcuoglu, P. Kohli, D. Hassabis, Highly accurate protein structure prediction with AlphaFold. Nature 596(7873), 583-589 (2021).

43. L. A. Wurmthaler, M. Sack, K. Gense, J. S. Hartig, M. Gamerdinger, A tetracyclinedependent ribozyme switch allows conditional induction of gene expression in Caenorhabditis elegans. Nat. Commun. 10(1), 491 (2019).

44. J. P. T. Ouyang, A. Folkmann, L. Bernard, C.-Y. Lee, U. Seroussi, A. G. Charlesworth, J. M. Claycomb, G. Seydoux, P granules protect RNA interference genes from silencing by piRNAs. Dev. Cell 50(6), 716-728.e6 (2019).

45. A. E. Dodson, S. Kennedy, Germ granules coordinate RNA-based epigenetic inheritance pathways. Dev. Cell 50(6), 704-715.e4 (2019).

46. D. Zhang, S. Tu, M. Stubna, W.-S. Wu, W.-C. Huang, Z. Weng, H.-C. Lee, The piRNA targeting rules and the resistance to piRNA silencing in endogenous genes. Science 359(6375), 587-592 (2018).

47. S. Devanapally, P. Raman, M. Chey, S. Allgood, F. Ettefa, M. Diop, Y. Lin, Y. E. Cho, A. M. Jose, Mating can initiate stable RNA silencing that overcomes epigenetic recovery. Nat. Commun. 12(1), 4239 (2021).

48. P. G. Okkema, S. W. Harrison, V. Plunger, A. Aryana, A. Fire, Sequence requirements for myosin gene expression and regulation in Caenorhabditis elegans. Genetics 135(2), 385-404 (1993). 
49. M. M. Crane, B. Sands, C. Battaglia, B. Johnson, S. Yun, M. Kaeberlein, R. Brent, A. Mendenhall, In vivo measurements reveal a single 5'-intron is sufficient to increase protein expression level in Caenorhabditis elegans. Sci. Rep. 9(1), 9192 (2019).

50. A. G. Charlesworth, U. Seroussi, N. J. Lehrbach, M. S. Renaud, A. E. Sundby, R. I. Molnar, R. X. Lao, A. R. Willis, J. R. Woock, M. J. Aber, A. J. Diao, A. W. Reinke, G. Ruvkun, J. M. Claycomb, Two isoforms of the essential C. elegans Argonaute CSR-1 differentially regulate sperm and oocyte fertility. Nucleic Acids Res., gkab619 (2021).

51. M. Placentino, A. M. J. Domingues, J. Schreier, S. Dietz, S. Hellmann, B. F. de Albuquerque, F. Butter, R. F. Ketting, Intrinsically disordered protein PID-2 modulates Z granules and is required for heritable piRNA-induced silencing in the Caenorhabditis elegans embryo. EMBO J.40(3), e105280 (2021).

52. I. F. Price, H. L. Hertz, B. Pastore, J. Wagner, W. Tang, Proximity labeling identifies LOTUS domain proteins that promote the formation of perinuclear germ granules in C. elegans bioRxiv 2021.07.27.453989 (2021); doi: https://doi.org/10.1101/2021.07.27.453989

53. N. Kalinava, J. Z. Ni, Z. Gajic, M. Kim, H. Ushakov, S. G. Gu, C. elegans heterochromatin factor SET-32 plays an essential role in transgenerational establishment of nuclear RNAimediated epigenetic silencing. Cell Rep. 25, 2273-2284.e3 (2018).

54. C. A. Spike, J. Bader, V. Reinke, S. Strome, DEPS-1 promotes P-granule assembly and RNA interference in C. elegans germ cells. Development 135(5), 983-993 (2008).

55. K. M. Suen, F. Braukmann, R. Butler, D. Bensaddek, A. Akay, C.-C. Lin, D. Milonaitytè, N. Doshi, A. Sapetschnig, A. Lamond, J. E. Ladbury, E. A. Miska, DEPS-1 is required for piRNAdependent silencing and PIWI condensate organisation in Caenorhabditis elegans. Nat.

Commun. 11(1), 4242 (2020).

56. I. Melentijevic, M. L. Toth, M. L. Arnold, R. J. Guasp, G. Harinath, K. C. Nguyen, D. Taub, J. A. Parker, C. Neri, C. V. Gabel, D. H. Hall, M. Driscoll, C. elegans neurons jettison protein aggregates and mitochondria under neurotoxic stress. Nature 542(7641), 367-371 (2017).

57. K. Tielking, S. Fischer, K. T. Preissner, P. Vajkoczy, R. Xu, Extracellular RNA in central nervous system pathologies. Front. Mol. Neurosci. 12, 254 (2019).

58. W. Li, K. S. Koutmou, D. J. Leahy, M. Li, Systemic RNA interference deficiency-1 (SID-1) extracellular domain selectively binds long double-stranded RNA and is required for RNA transport by SID-1. J. Biol. Chem. 290(31), 18904-18913 (2015).

59. M. C. Ow, K. Borziak, A. M. Nichitean, S. Dorus, S. E. Hall, Early experiences mediate distinct adult gene expression and reproductive programs in Caenorhabditis elegans. PLoS Genet. 14, e1007219 (2018). 
60. P. Kadekar, R. Roy, AMPK regulates germline stem cell quiescence and integrity through an endogenous small RNA pathway. PLoS Biol. 17, e3000309 (2019).

61. M. F. Palominos, L. Verdugo, C. Gabaldon, B. Pollak, J. Ortiz-Severin, M. A. Varas, F. P. Chávez, A. Calixto, Transgenerational diapause as an avoidance strategy against bacterial pathogens in Caenorhabditis elegans. mBio 8, pii: e01234-17 (2017).

62. R. S. Moore, R. Kaletsky, C. T. Murphy, Piwi/PRG-1 Argonaute and TGF- $\beta$ mediate transgenerational learned pathogenic avoidance. Cell 177(7), 1827-1841 (2019).

63. R. Kaletsky, R. S. Moore, G. D. Vrla, L. R. Parsons, Z. Gitai, C. T. Murphy, C. elegans interprets bacterial non-coding RNAs to learn pathogenic avoidance. Nature 586(7829), 445-451 (2020).

64. R. Posner, I. A. Toker, O. Antonova, E. Star, S. Anava, E. Azmon, M. Hendricks, S. Bracha, H. Gingold, O. Rechavi, Neuronal small RNAs control behavior transgenerationally. Cell 177, 1814-1826.e15 (2019).

65. H. Ohno, Z. Bao, Small RNAs couple embryonic developmental programs to gut microbes. bioRxiv: 10.1101/2020/11/13/381830 (2020).

66. A. M. Jose, Heritable epigenetic changes alter transgenerational waveforms maintained by cycling stores of information. Bioessays 42(7), e1900254 (2020).

67. M. Chey, A. M. Jose, Heritable epigenetic changes at single genes: challenges and opportunities in Caenorhabditis elegans. Trends Genet., in press (2021); doi: https://doi.org/10.1016/j.tig.2021.08.011

68. S. Brenner, The genetics of Caenorhabditis elegans. Genetics 77(1), 71-94 (1974).

69. C. C. Mello, J. M. Kramer, D. Stinchcomb, V. Ambros, Efficient gene transfer of C. elegans: extrachromosomal maintenance and integration of transforming sequences. EMBO J. 10(12), 3959-3970 (1991).

70. C. Frøkjær-Jensen, M. W. Davis, M. Ailion, E. M. Jorgensen, Improved Mos1-mediated transgenesis in C. elegans. Nat. Methods 9, 117-118 (2012).

71. A. M. Jose, G. A. Garcia, C. P. Hunter, Two classes of silencing RNAs move between $C$. elegans tissues. Nat. Struct. Mol. Biol. 18(11), 1184-1188 (2011).

72. P. Raman, S. M. Zaghab, E. C. Traver, A. M. Jose, The double-stranded RNA binding protein RDE-4 can act cell autonomously during feeding RNAi in C.elegans. Nucleic Acids Res. 45(14), 8463-8473 (2017). 
73. E. Kage-Nakadai, R. Imae, Y. Suehiro, S. Yoshina, S. Hori, S. Mitani, A conditional knockout toolkit for Caenorhabditis elegans based on the Cre/loxP recombination. PLoS One $\mathbf{9}$, e114680 (2014).

74. J. A. Arribere, R. T. Bell, B. X. H. Fu, K. L. Artiles, P. S. Hartman, A. Z. Fire, Efficient marker-free recovery of custom genetic modifications with CRISPR/Cas9 in Caenorhabditis elegans. Genetics 198(3), 837-846 (2014).

75. A. Paix, A. Folkmann, D. Rasoloson, G. Seydoux, High efficiency, homology-directed genome editing in Caenorhabditis elegans using CRISPR-Cas9 ribonucleoprotein complexes. Genetics 201(1), 47-54 (2015).

76. G. A. Dokshin, K. S. Ghanta, K. M. Piscopo, C. C. Mello, Robust genome editing with short single-stranded and long, partially single-stranded DNA donors in Caenorhabditis elegans. Genetics 210(3), 781-787 (2018).

77. H. Tabara, M. Sarkissian, W. G. Kelly, J. Fleenor, A. Grishok, L. Timmons, A. Fire, C. C. Mello, The $r d e-1$ gene, RNA interference, and transposon silencing in C.elegans. Cell 99(2), 123-132 (1999).

78. J. Vicencio, C. Martínez-Fernández, X. Serrat, J. Cerón, Efficient generation of endogenous fluorescent reporters by nested CRISPR in Caenorhabditis elegans. Genetics 211(4), 1143-1154 (2019).

79. A. M. Jose, Y. A. Kim, S. Leal-Ekman, C. P. Hunter, Conserved tyrosine kinase promotes the import of silencing RNA into Caenorhabditis elegans cells. Proc. Natl. Acad. Sci. 109(36), 14520-14525 (2012).

80. A. D. Levy, J. Yang, J. M. Kramer, Molecular and genetic analyses of the Caenorhabditis elegans dpy-2 and dpy-10 collagen genes: a variety of molecular alterations affect organismal morphology. Mol Biol Cell 4(8), 803-817 (1993).

81. J. Schindelin, I. Arganda-Carreras, E. Frise, V. Kaynig, M. Longair, T. Pietzsch, S. Preibisch, C. Rueden, S. Saalfeld, B. Schmid, J.-Y. Tinevez, D. J. White, V. Hartenstein, K. Eliceiri, P. Tomancak, A. Cardona, Fiji: an open-source platform for biological-image analysis. Nat. Methods 9, 676-682 (2012).

82. C. J. Harris, A. Molnar, S. Y. Müller, D. C. Baulcombe, FDF-PAGE: a powerful technique revealing previously undetected small RNAs sequestered by complementary transcripts. Nucleic Acids Res. 43, 7590-7599 (2015).

83. M. Martin, Cutadapt removes adapter sequences from high-throughput sequencing reads. EMBnet.journal 17(1), 10-12 (2011).

84. R. Patro, G. Duggal, M. I. Love, R. A. Irizarry, C. Kingsford, Salmon provides fast and biasaware quantification of transcript expression. Nat. Methods 14, 417-419 (2017). 
85. C. Soneson, M. I. Love, M. D. Robinson, Differential analyses for RNA-seq: transcript-level estimates improve gene-level inferences. F1000Research 4, doi: 10.12688/f1000research.7563.1 (2015).

86. M. D. Robinson, A. Oshlack, A scaling normalization method for differential expression analysis of RNA-seq data. Genome Biol. 11(3), R25 (2010).

87. C. W. Law, Y. Chen, W. Shi, G. K. Smyth, voom: precision weights unlock linear model analysis tools for RNA-seq read counts. Genome Biol. 15, R29 (2014).

88. K. J. Reed, J. M. Svendsen, K. C. Brown, B. E. Montgomery, T. N. Marks, T. Vijayasarathy, D. M. Parker, E. O. Nishimura, D. L. Updike, T. A. Montgomery, Widespread roles for piRNAs and WAGO-class siRNAs in shaping the germline transcriptome of Caenorhabditis elegans. Nucleic Acids Res. 48(4), 1811-1827 (2020).

89. D. Kim, J. M. Paggi, C. Park, C. Bennett, S. L. Salzberg, Graph-based genome alignment and genotyping with HISAT2 and HISAT-genotype. Nat. Biotechnol. 37, 907-915 (2019).

90. H. Li, B. Handsaker, A. Wysoker, T. Fennell, J. Ruan, N. Homer, G. Marth, G. Abecasis, R. Durbin, 1000 Genome Project Data Processing Subgroup, The Sequence Alignment/Map format and SAMtools. Bioinformatics 25(16), 2078-2079 (2009).

91. F. Madeira, Y. M. Park, J. Lee, N. Buso, T. Gur, N. Madhusoodanan, P. Basutkar, A. R. N. Tivey, S. C. Potter, R. D. Finn, R. Lopez, The EMBL-EBI search and sequence analysis tools APIs in 2019. Nucleic Acids Res. 47(W1), W636-W641 (2019).

92. N. J. Bowen, J. F. McDonald, Genomic analysis of Caenorhabditis elegans reveals ancient families of retroviral-like elements. Genome Res 9(10), 924-935 (1999). 


\section{Figures and Legends:}

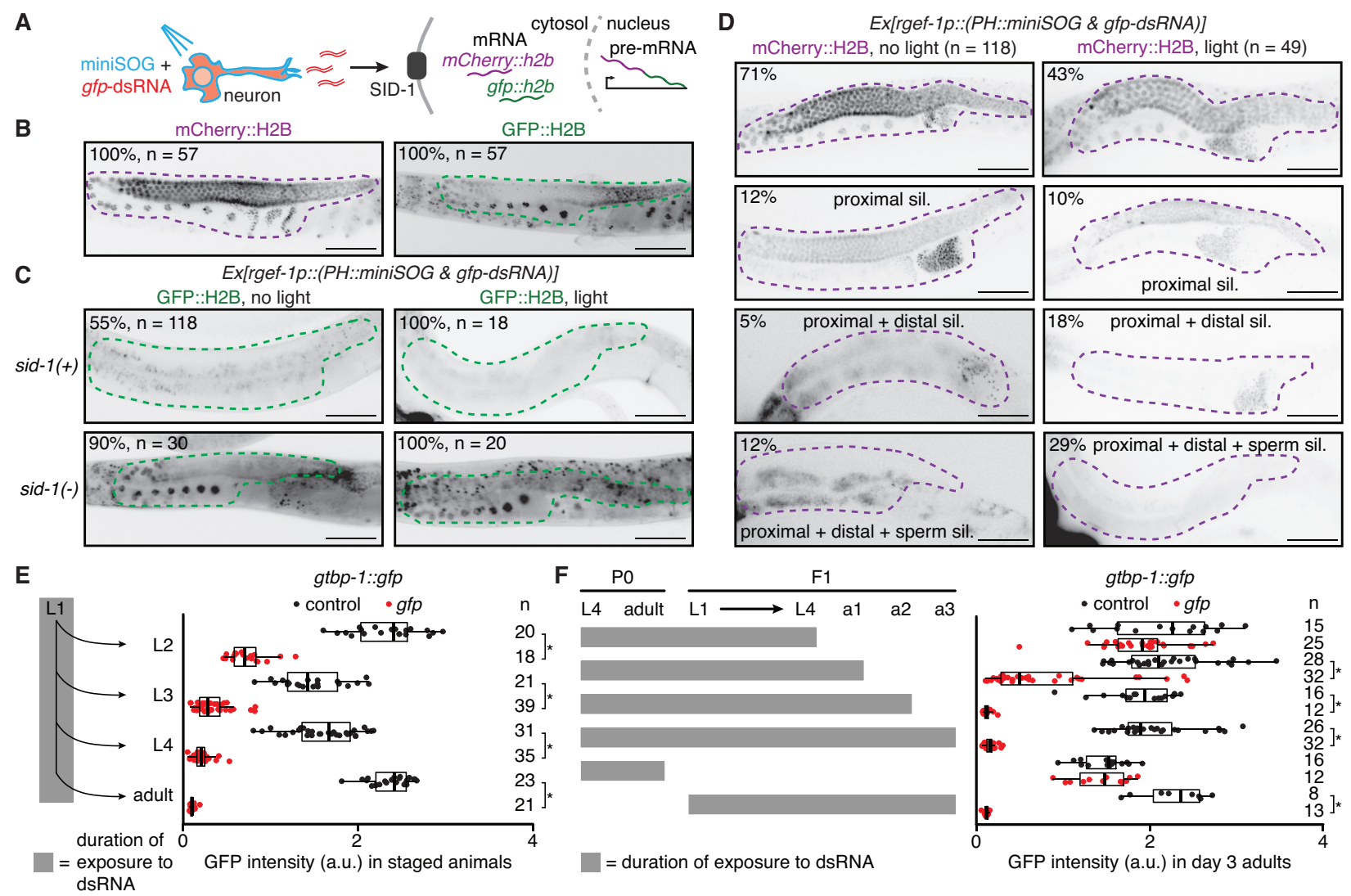

Fig. 1. Timed delivery of neuronal or ingested dsRNA suggests spatiotemporal differences

in germline entry. (A) Schematic illustrating exposure of animals expressing a singlet oxygen generator (miniSOG) and $g f p$-dsRNA in neurons to blue light and subsequent release of dsRNA. Such extracellular dsRNA can enter the germline through the dsRNA importer SID-1 and silence $g f p:: h 2 b$ mRNA from a two-gene operon that expresses $m C h e r r y:: h 2 b$ and $g f p:: h 2 b$ as part of a single pre-mRNA. (B, C , and D) Images of single gonad arms in adult animals with the two-gene

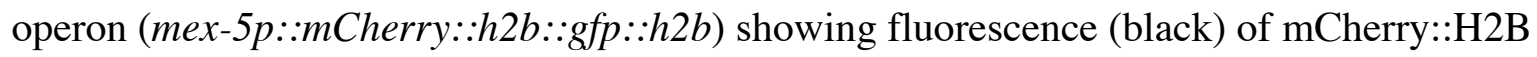
(magenta outline) or of GFP::H2B (green outline). Punctate autofluorescence from the intestine can also be seen. Numbers of animals assayed (n) and percentages of adult animals with the depicted expression patterns are indicated. Scale bars, $50 \mu \mathrm{m}$. (B) mCherry::H2B fluorescence is seen throughout the germline (left) and GFP::H2B fluorescence is seen in the oocytes and in the 
distal gonad (right). (C) GFP::H2B fluorescence in sid-1(+) and sid-1(-) animals expressing membrane-localized miniSOG (PH::miniSOG) and $g f p$-dsRNA driven by a neuronal promoter (rgef-1p) from a multi-copy transgene (Ex,jamEx214) without (left) or with (right) exposure to blue light at 48 hours post L4-stage of parent. (D) mCherry::H2B fluorescence in sid-1(+) animals with the transgene Ex. Silencing of mCherry is enhanced in the distal gonad (third row) and sperm (fourth row) after exposing animals to blue light at 48 hours and 54 hours post L4stage of parent. Also see Supplementary Fig. S1 and Supplementary Fig. S2. (E) Silencing in the germline after continuous exposure of $g t b p-1:: g f p$ animals to bacteria expressing dsRNA starting at the L1 stage, and imaging of separate cohorts at each subsequent stage. (left) Schematic of assay. (right) GFP intensity (a.u.) in $g t b p-1: \because g f p$ animals at indicated stages quantified in germ cells (larvae) or eggs in utero (adults) after exposure to control (black) or $g f p$-dsRNA (red). The numbers of animals scored at each stage (n) are depicted. (F) Schematic depicting duration of exposure for different cohorts of P0 and F1 animals to bacteria expressing dsRNA (left) and quantification of GFP intensity (a.u.) as in (E) in F1 animals on the third day of adulthood (right). The numbers of adult day $3 \mathrm{~F} 1$ animals scored (n) are depicted. Asterisks in (E) and (F) indicate $P<0.05$ with Bonferroni correction using Mann-Whitney $\mathrm{U}$ test for two-sided comparisons between animals exposed to control or $g f p$-dsRNA. Also see Supplementary Fig. S3. 


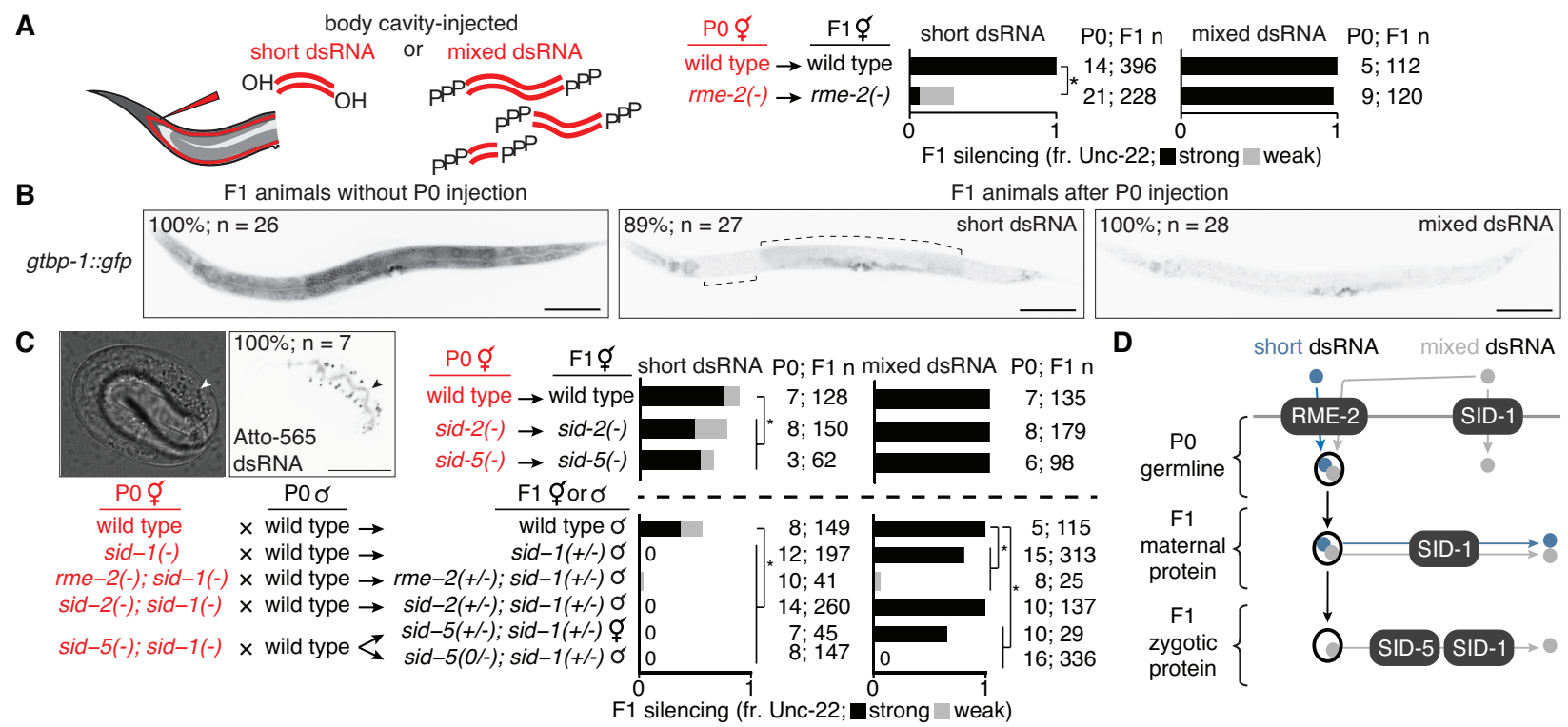

Fig. 2. Transport of dsRNA from parental circulation to progeny occurs through two

routes with distinct substrate selectivity. (A) Hermaphrodite animals of indicated genotypes

(in red) were injected in the body cavity with 50-bp unc-22-dsRNA synthesized with a 5'-OH

( short dsRNA, left bars) or unc-22-dsRNA with a 5'-triphosphate transcribed from a $\sim 1.1 \mathrm{~kb}$

template (mixed dsRNA, right bars). Hermaphrodite self-progeny of injected animals were

scored for unc-22 silencing (fr. Unc-22: strong, black; weak, grey). Numbers of injected parents

and scored progeny (P0; F1 n) are indicated. Also see Supplementary Fig. S2 and Supplementary

Fig. S4. (B) Fluorescence images of progeny from animals with a $g f p$ tag of the ubiquitously

expressed gene $g t b p-1(g t b p-1: \because g f p)$ that were not injected (left), injected with 50-bp gfp-dsRNA

(short dsRNA injection, middle), or injected with dsRNA transcribed from a 730-bp template

(mixed dsRNA injection, right). Complete silencing is not observed in neurons or in the

developing vulva; brackets indicate additional regions with dim GFP fluorescence. Numbers of

animals assayed (n) and percentages of L4-staged animals with the depicted expression patterns

are indicated. Scale bar, $100 \mu \mathrm{m}$. Also see Supplementary Fig. S5. (C) Requirements for

intergenerational transport of extracellular dsRNA. (top left) Differential Interference Contrast 
(DIC) and fluorescence images of a developing embryo from an animal injected in the body cavity with 50-bp dsRNA of the same sequence as in (B) and labeled at the 5' end of the antisense strand with Atto-565. Accumulation within the intestinal lumen (arrowhead), number of embryos imaged (n), and percentage of embryos with depicted pattern of fluorescence are indicated. Scale bar, $20 \mu \mathrm{m}$. (top right and bottom) Hermaphrodite animals of the indicated genotypes were injected with short dsRNA (left bars) or mixed dsRNA (right bars) and selfprogeny (top right) or cross progeny after mating with wild-type males (bottom) were analyzed as in (A). Cases of no observable silencing are indicated with '0'. (D) Schematic summarizing requirements for transport of dsRNA from parental circulation to developing progeny. Asterisks indicate $P<0.05$ with Bonferroni correction using $\chi^{2}$ test. 
A

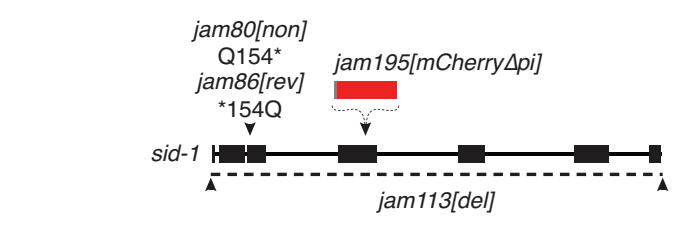

B

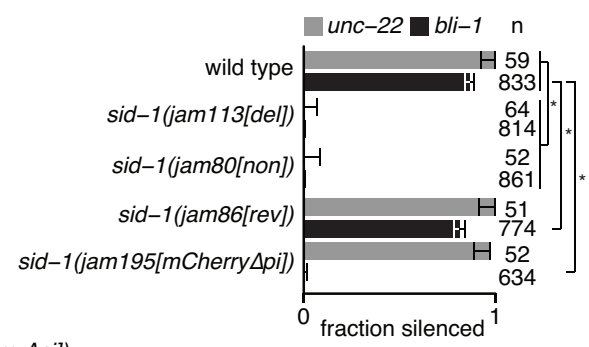

C $1 \mathrm{~kb}$
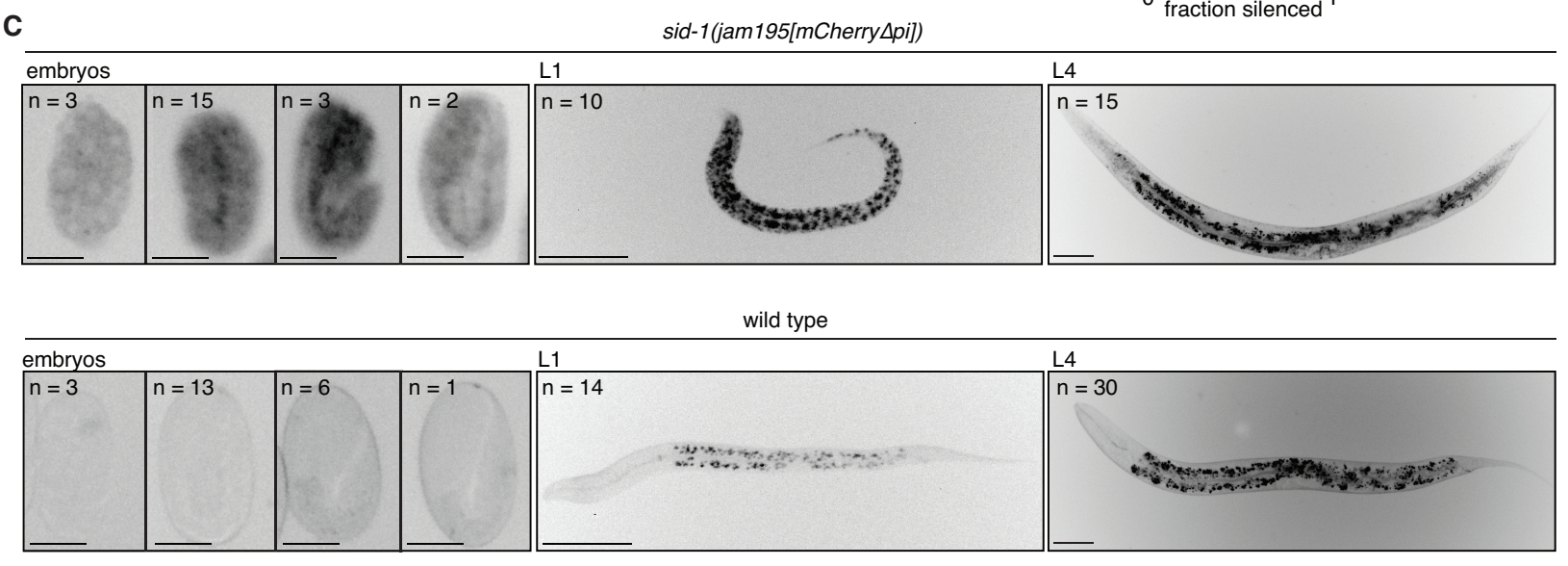

D
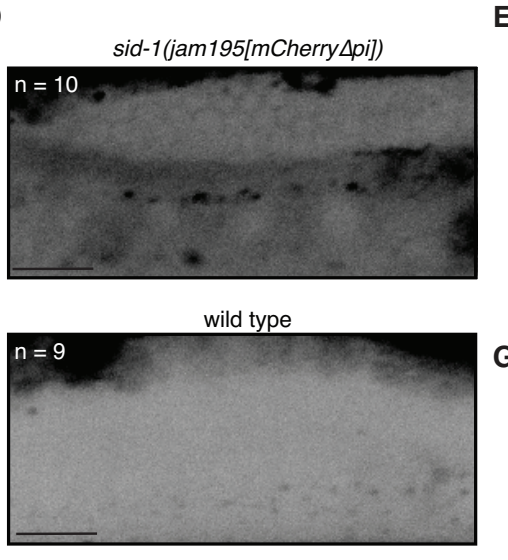

E
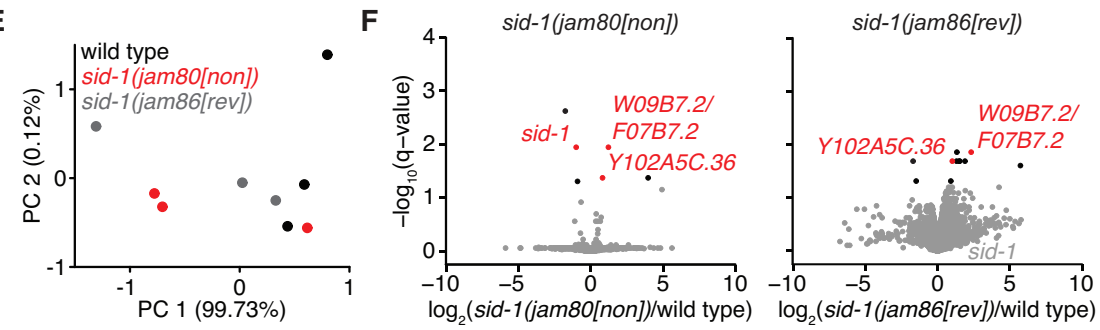

G

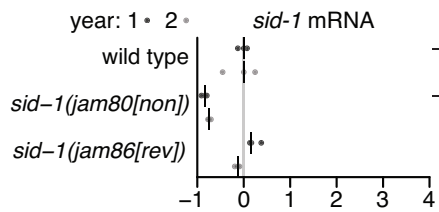

Y102A5C.36 mRNA

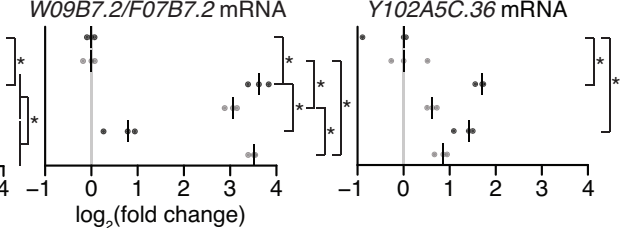

Fig. 3. Ancestral loss of the dsRNA importer SID-1 results in the accumulation of mRNAs

of two germline genes in wild-type descendants. (A) Schematic of modifications at the sid-1 gene generated using Cas9-mediated genome editing. Deletion of the entire coding sequence (jam113[del]), a nonsense mutation (jam80[non]), its reversion to wild-type sequence (jam86[rev]), and insertion of $m$ Cherry sequence that lacks piRNA binding sites (46-47) (jam195[mCherry $\Delta$ pi]) are depicted. (B) Fractions of animals of the indicated genotypes that show silencing in response to $u n c$-22-dsRNA (grey) or bli-1-dsRNA (black) are plotted. Tagging 
SID-1 with mCherry (sid-1(jam195[mCherry $\Delta p i]))$ likely results in a partially functional SID-

$1::$ mCherry fusion protein because $u n c-22$ silencing is robust but bli- 1 silencing is very weak

(only 6 of 634 animals showed the Bli-1 defect). Numbers of animals scored (n), significant differences using two-tailed test with Wilson's estimates for single proportions (asterisks, $P<$ 0.05 with Bonferroni correction) and 95\% CI (error bars) are indicated. (C and D) Representative images showing fluorescence from SID-1::mCherry (black) in (C) developing embryos (left), L1stage (middle), L4-stage (right) or (D) adult gonad arms of sid-1(jam195[mCherry Api]) animals (top) compared to no detectable fluorescence in wild-type animals of the same stages (bottom). Numbers of (C) embryos of each stage, L1 animals, L4 animals, and (D) adult gonad arms imaged (n) are depicted and $100 \%$ of animals exhibited the depicted expression patterns. For animals imaged in (D), the distal germline was obstructed by the intestine in 1/10 sid-

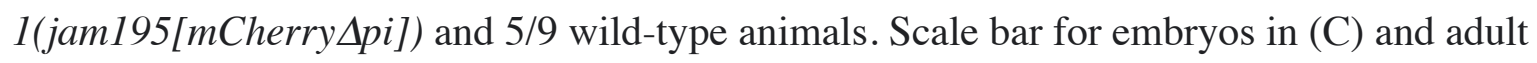
gonad arms in (D), $20 \mu \mathrm{m}$; scale bar for larvae in (C), $50 \mu \mathrm{m}$. Also see Supplementary Fig. S6. (E) Principal components explaining the variance between wild type (black), sid-1(jam80[non]) (red), and sid-1(jam86[rev]) (grey) polyA+ RNA samples. Almost all of the variance between samples is explained by PC 1. (F) Volcano plots of changes in the abundance of polyA+ RNA in sid-1(jam80[non]) (left) and sid-1(jam86[rev]) (right) animals compared with wild-type animals (black, $q<0.05$; red, $q<0.05$ and with change in the same direction in sid-1(jam80[non]) and sid-1(jam113[del]); see Supplementary Fig. S9). While sid-1 transcript levels in sid1(jam86[rev]) are comparable to that in wild type (grey), W09B7.2/F07B7.2 and Y102A5C.36 transcript levels remain elevated in sid-1(jam86[rev]) (red). (G) Levels of spliced sid-1, W09B7.2/F07B7.2 and Y102A5C.36 transcripts measured using RT-qPCR. The median of three technical replicates is plotted for each of three biological replicates (bar indicates median) 
bioRxiv preprint doi: https://doi.org/10.1101/2021.10.05.463267; this version posted October 6, 2021. The copyright holder for this preprint

(which was not certified by peer review) is the author/funder, who has granted bioRxiv a license to display the preprint in perpetuity. It is made available under aCC-BY-NC-ND 4.0 International license.

assayed one year apart (year 1, dark grey; year 2, light grey). Asterisks indicate $P<0.05$ with

Bonferroni correction using two-tailed Student's t-test. 

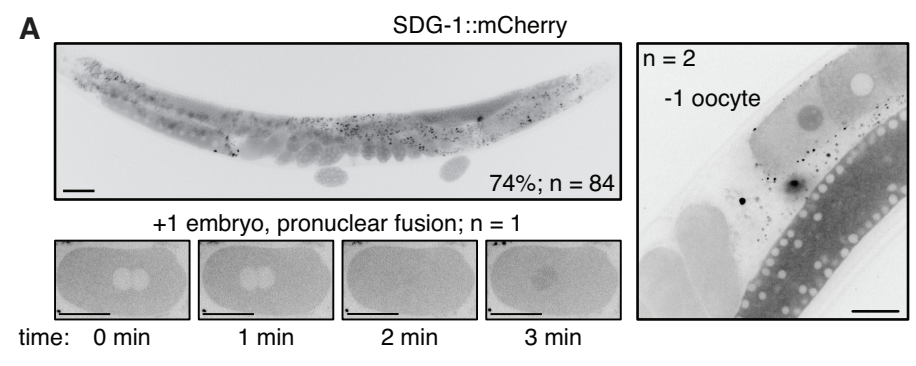

C anterior arm .

B
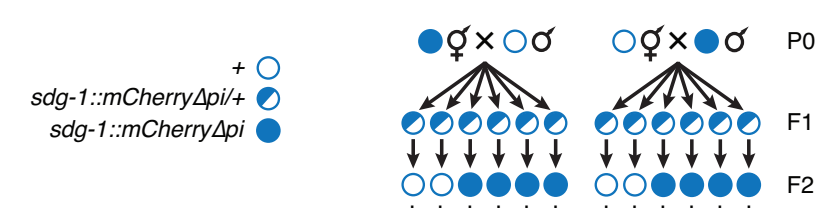

sdg-1::mCherry $\Delta$ pi

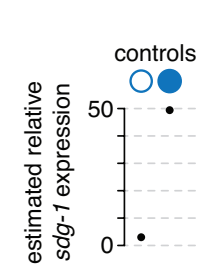

D

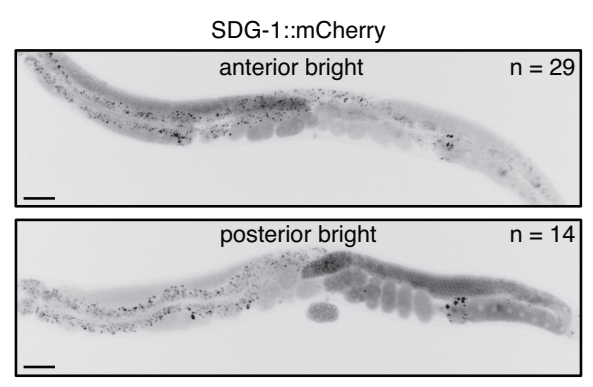

E

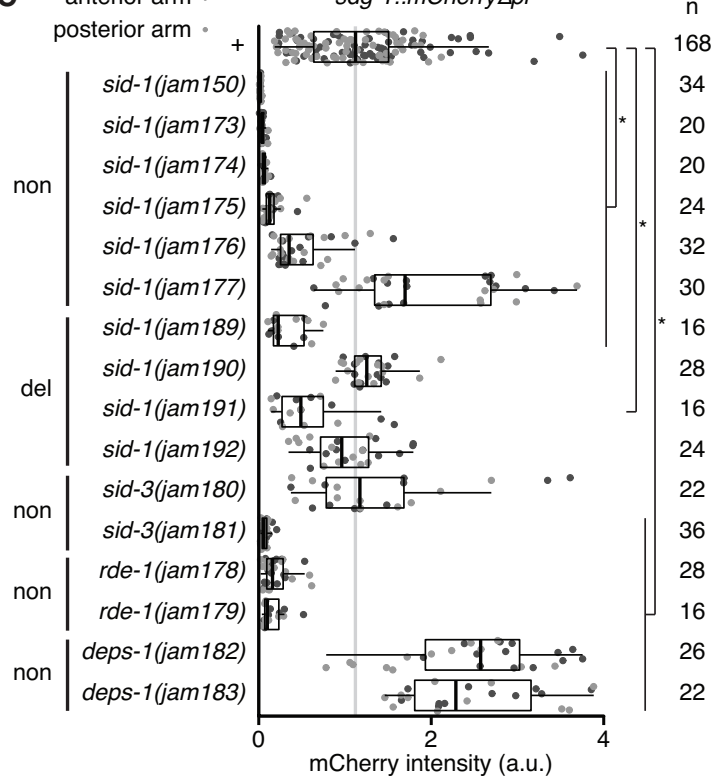

anterior arm .

posterior arm - sdg-1::mCherry $\Delta p i \quad n$

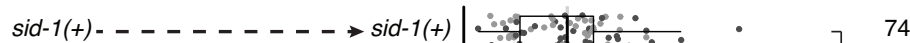

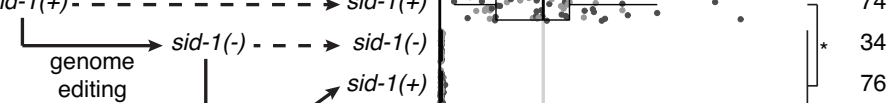

editing

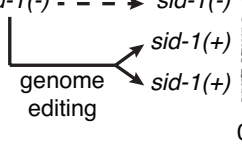

mCherry intensity (a.u.)

Fig. 4. A SID-1-dependent gene is prone to stochastic changes in gene expression that can

become heritable. (A) Fluorescence images of SDG-1::mCherry in adult animals. Numbers of animals assayed (n) and percentages of adult animals with the depicted expression patterns (top left) are indicated. Punctate fluorescence in the intestine likely represents autofluorescence. Scale bars, $50 \mu \mathrm{m}$ (top left) or $20 \mu \mathrm{m}$ (right and bottom left). (top left) Cytoplasmic fluorescence is detectable throughout the germline and in embryos. (right) Nuclear localization of SDG1::mCherry in the -1 oocyte was detected in confocal imaging of two animals. (bottom left) An embryo undergoing pronuclear fusion after fertilization showed dynamic nuclear localization before the first cell division (time in minutes). (B) Lineages and estimated relative $s d g-1$ expression 10 generations after mating wild-type (open circle) males and $s d g-1:: m C h e r r y \Delta p i$ (filled circle) hermaphrodites and vice versa and isolating $s d g-1(+)$ or $s d g-1:: m C h e r r y$ animals 
from F1 heterozygotes (half-filled circle). Expression of $s d g-1$ in the F10 generation was measured by RT-qPCR of $s d g-1$ mRNA purified from pooled wild-type animals of mixed stages or by quantification of SDG-1::mCherry fluorescence in gonad arms of adult $s d g-1:: m C h e r r y \Delta p i$ animals. Relative levels of $s d g-1$ mRNA and SDG-1::mCherry fluorescence intensity were converted to units of estimated relative $s d g-1$ expression (see Materials and methods) for comparison. See Supplementary Fig. S13 for raw data. (C) Fluorescence intensity measurements (arbitrary units, a.u.) quantified as in (B) (anterior gonad arm, light grey; posterior gonad arm, dark grey) in adult animals with $s d g-1:: m$ Cherry $\Delta p i(+)$ and additionally with mutations in single genes that regulate dsRNA import (sid-1 or sid-3) or RNA silencing ( $r d e-1$ or deps-1) are shown. Nonsense mutations (non) or deletions (del) introduced by genome editing and numbers of gonad arms (n) quantified for each isolate are indicated. Asterisks indicate $P<0.05$ with Bonferroni correction using Mann-Whitney U test for two-sided comparisons between animals with $s d g-1:: m C h e r r y \Delta p i(+)$ and animals with additional mutations. (D) Representative images showing asymmetric fluorescence of SDG-1::mCherry (black) with bright anterior (top) or bright posterior (bottom) gonad arms. Animals with at least one gonad arm brighter than the dimmest wild-type gonad arm in (C) and with >2-fold difference in fluorescence between both gonad arms were selected as having asymmetric fluorescence (anterior bright $(\mathrm{n}=29)$ : wild type $(+)-$ 17/84, sid-1(-) - 5/122, sid-3(-) - 1/29, rde-1(-) - 2/22, deps-1(-) - 4/24, and posterior bright (n = 14): wild type $(+)-5 / 84$, sid-1(-) -6/122, rde-1(-) - 2/22, deps-1(-) - 1/24). Mutations in genes required for dsRNA import or subsequent silencing resulted in fewer animals with asymmetric fluorescence between gonad arms (a combined proportion of 21/197 for sid-1, sid-3, rde- 1 and deps- 1 mutants versus 22/84 for wild type, $P=0.0009$ using two-tailed test with Wilson's estimates for single proportions). Punctate fluorescence in the intestine likely 
represents autofluorescence. Scale bar, $50 \mu \mathrm{m}$. (E) Animals with sdg-1::mCherry $\Delta p i$ that show altered fluorescence upon loss of sid-1 remain changed despite reversion of sid-1 nonsense mutation to wild-type sequence. Quantification of SDG-1::mCherry in adult gonad arms as in (B). Also see Supplementary Fig. S12 and S14. 